\title{
ESTIMATIVA DA PREVALÊNCIA DA HANSENÍASE PELA INVESTIGAÇÃO EM DEMANDA INESPECÍFICA DE AGÊNCIAS DE SAÚDE*
}

\author{
Diltor Vladimir Opromolla* \\ Rubens Cunha Nobrega*** \\ Neusa Nunes da Silva e Gonçalves*** \\ Silvia Helena Pereira Padovani*** \\ Carlos Roberto Padovani*** \\ Aguinaldo Gonçalves***
}

OPROMOLLA, D. V. et al. Estimativa da prevalência da hanseníase pela investigação em demanda inespecifica de agências de saúde. Rev. Saúde públ., S.Paulo, 24: 178-85, 1990.

RESUMO: Dada a importância das taxas de prevalência e incidência de uma moléstia para conhecimento de seu comportamento e planejamento de seu controle, em nível do coletivo, procedeu-se a estudo na cidade de Taubaté, Vale do Paraíba, SP (Brasil) da ocorrência da hanseníase em clientela de agências de saúde com o objetivo de explorar o emprego desta metodologia para estimar o "iceberg" epidemiológico da doença (i.e., o número total de casos, incluindo os existentes, mas não oficialmente registrados). Foram averiguados clientes com idade igual ou superior a quinze anos, independentemente de variáveis pessoais ( como sexo, idade, condiçăo social, estado civil), pois admitiu-se ser conhecida a distribuição populacional da doença e as características dos serviços. Consideradas as peculiaridades locais (v.g. identidade das diferentes clientelas, disponibilidade de consultórios, horários de maior fluxo), o processo de amostragem utilizado foi o da casualizaçăo simples. Os doentes detectados em atividade, $40 \mathrm{em}$ 10.013 pessoas examinadas, corresponderam ao indice de prevalência de $3,99 / 1.000$, com intervalo de $\infty n-$ fiança (ao nível de $5 \%$ de confiabilidade) variando de 3.365 a $4.625 / 1.000$, o que significa que o acréscimo mínimo estimado da prevalência é da ordem de $52 \%$ e máximo de $109 \%$. Houve predomínio, entre os doentes, da forma indeterminada $(35,0 \%)$ mais explicitamente quando são eles estratificados em registrados os casos novos: nesta categoria, a forma indeterminada atingiu $56,5 \%$. Sua distribuiçăo por faixa etária não se distanciou da observada com os dados de registro oficial, segundo se constatou pelo cotejo dos casos observados e esperados, calculados a partir dos indicadores populacionais; quanto à proporção sexual, registrou-se predomínio de elementos do sexo masculino.

DESCRITORES: Hanseníase, epidemiologia. Serviços de saúde.

\section{INTRODUÇÃO}

Como sabido, prevalência e incidência são indicadores básicos para o conhecimento e manejo de qualquer doença em saúde coletiva. No primeiro sentido, seus valores dimensionam a magnitude da mesma quanto a constituir-se, ou não, problema de saúde pública; no segundo, săo fundamentos dos procedimentos de previsão e administração sanitária de recursos humanos e materiais, de custeio e de capital. No entanto, em países do Terceiro Mundo săo muito imprecisos em decorrência às limitaçōes na obtenção, registro, processamento e análise de informaçoes de saúde.

Em relação à hanseníase, a questão é ainda mais complexa. Dado seu longo período de incu- bação, até a expressão "incidência", não é rigorosamente adequada, pois não existem casos novos em apenas um ano, mas casos detectados no período, sejam novos ou não (Lechat e col. ${ }^{15}, 1986$ ). Mais grave, no entanto é o fenômeno conhecido pelos hansenólogos como "iceberg epidemiológico", pelo qual se designa o fato de que o número de casos conhecidos da doença é sempre apenas a extremidade visivel de contingente muito mais numeroso (Gonçalves ${ }^{10}$, 1987).

Quanto é esta diferença do real para o visível e como conhecê-la são, portanto, questões essenciais. Noordeen e Lopes Bravo ${ }^{18}$ (1986), para 5.368.202 doentes registrados, no mundo, estimam a existência de dez a doze milhões de casos, pois, segundo Lechat ${ }^{13}$ (1983), em algumas regiōes o número de Realizado com apoio financeiro do Conselho Nacional de Desenvolvimento Científico e Tecnológico (CNPq). Processo $n^{\circ} 300379 / 87$

"* Centro de Estudos "Reynaldo Quagliato", Hospital de Dermatologia Sanitária "Lauro de Souza Lima" - Estrada Bauru-Jaú, km 115 - 17100 - Bauru, SP - Brasil.

* * Bolsista do Conselho Nacional de Desenvolvimento Científico e Tecnológico. 
pacientes năo detectados aparentemente excede 0 sob tratamento. A este propósito, Bechelli e Martinez Dominguez ${ }^{3}$ (1966) propuseram métodos para estimar os casos não detectados de hanseníase, baseados em dados de Grupo Consultor da Organização Mundial da Saúde: se o programa de controle da endemia em dada região, segundo tais métodos, se revela satisfatório, regular ou precário, adiciona-se fator corretivo ao número de casos registrados, respectivamente de $75 \%, 150 \%$ e $300 \%$.

Uma concepção corrente consiste em pressupor que tal diferença possa ser conhecida com maior precisão, comparando-se os números oficiais da demanda passiva às agências de saúde com os obtidos em censos intensivos ou em inquéritos hansenológicos, entre escolares ou familiares de doentes, por exemplo. A primeira situação mencionada é aquela onde toda a população é examinada metodicamente; no entanto, a baixa ocorrência da hanseníase torna tal estudo proibitivamente caro, em tempo, custo e pessoal, e gera problemas consideráveis em logística, supervisão e manejo de dados (Fine ${ }^{8}, 1981$ ); o perfil epidemiológico, ademais, é radicalmente oposto ao habitual, "com reduzida porcentagem de lepromatosos (em torno de $10 \%$ ), pertencendo a maioria dos doentes despistados a lepra tuberculóide e, em menor número a indeterminada" (Bechelli e Curban ${ }^{2}, 1988$ ).

Quanto ao inquérito em escolares, Ganapati 9 (1983) aplicou-o em comunidade urbana em que a hanseníase é endèmica, obtendo resultados indicativos de que, embora econômico, não resultou na identificação de um número significativamente maior de casos na comunidade, seja em valor absoluto, seja em proporção de casos infectantes.

Neste sentido já Bechelli e col. ${ }^{4}$ (1973), revendo relatos de prevalência em crianças e na população geral em diferentes continentes, informaram que "parecia, de tais relatos, que as taxas encontradas entre pessoas de 5 a 14 anos de idade geralmente refletiam o grau da endemicidade: se esta taxa é baixa, baixa prevalência geral pode ser esperada; se alta, a última também o será (...), igualmente, em algumas situaçōes, pode sugerir a ordem de grandeza da prevalência total na área considerada".

Tendência contemporânea que vem sendo observada consiste no estudo de prevalência da doença através do exame exaustivo, não censitariamente nem em grupos específicos, mas de segmentos amostrais da população. A esse respeito Sundarcsan e Sansarricq ${ }^{26}$ (1982) formularam um manual metodológico introdutório. Aplicado este procedimento, resultados interessantes vêm sendo obtidos. Somse e col. ${ }^{25}$ (1985), por exemplo, em avaliação da endemia hansênica em coletividade da República Centro-Africana, concluíram serem questionáveis os dados anteriormente disponíveis que minimizavam de três a quatro vezes os parâmetros do comportamento do agravo na população.

Em síntese, segundo a Organização Mundial de Saúde (citado por OPS 21, 1983), para áreas de baixa endemicidade "o exame de contatos domiciliares de casos infecciosos, especialmente crianças, e de pessoas notificadas como casos suspeitos, constitui o método mais eficiente e prático de descobrimento dos casos; quando a prevalência for de aproximadamente 1 por 1000 ou superior, dever-seiam utilizar métodos adicionais, tais como o exame de crianças em idade escolar, de recrutas militares e de outros grupos selecionados; censos intensivos são aconselháveis para as populações hiperendêmicas".

Nesta linha de idéias e partindo da preocupação inicialmente mencionada de subsidiar o planejamento dos serviços de saúde, formulou-se e desenvolveu-se, pioneiramente, estudo da prevalência da hanseníase em clientela de agências de saúde, com o objetivo de, além de subsidiar esta metologia para estimar o "iceberg epidemiológico", prover informações operacionais a respeito para a gestão dos serviços de saúde. A presente comunicação apresenta e discute os resultados preliminares disponíveis. Uma vez testado o procedimento, ele poderá, a juízo das autoridades setoriais, ser expandido a outras regioes, permitindo, estritamente quanto aos aspectos epidemiológicos, maior exatidão dos indicadores epidemiométricos em uso. Ademais, encerra importante aspecto quanto à organização dos serviços de saúde, por constituir-se em pragmático instrumento de treinamento de recursos humanos nas unidades em que seja aplicado.

\section{MATERIAL E MÉTODOS}

As unidades sanitárias identificadas para a coleta de dados primários localizam-se em Taubaté-SP. A escolha desta cidade para estudo decorre do fato de que as características de operacionalização dos serviços aí existentes têm sido aparentemente menos influentes nos valores obtidos nos indicadores epidemiométricos que a própria realidade epidemiológica.

Não obstante a taxa de prevalência de hanseníase oficialmente registrada no município de Taubaté, em 1988, ser da ordem de 1,50/1000 para a população geral, estudo-piloto realizado por nosso grupo (Opromolla e col. ${ }^{20}, 1988$ ) revelou valor de 4,84/1000 em 1.856 pessoas com idade igual ou superior a 15 anos, amostradas na demanda espontânea de Unidades Sanitárias da cidade.

Pelas técnicas de amostragem (Cochran ${ }^{5}, 1965$ ), condicionando-se um nível de $5 \%$ de significância e $30 \%$ de precisão, e utilizando a taxa de prevalência obtida através de demanda às Unidades Sanitárias, obtém-se 8.760 como o tamanho amostral. 
Admitidas as peculiaridades de operacionalização locais (vg. características das diferentes clientelas, disponibilidades de consultórios e horários de maior fluxo), o processo de amostragem utilizado é o da casualização simples.

O critério de alocação adotado foi o de admitir clientes com idade superior a 15 anos, independentemente de variáveis epidemiológicas pessoais (como sexo, condição social, estado civil, escolaridade, faixa etária), considerando o conhecido em relaçăo a distribuição populacional da moléstia e às características do serviço.

Quanto à rede de serviços de saúde da cidade, embora esteja ela integrada ao processo geral por que passa o setor saúde no Estado e no País, no sentido de um sistema unificado pluri-institucional, as características históricas que levaram a origem anterior dos diversos orgãos ainda continuam vigindo. Desse modo, os dois Centros de Saúde, incluídos na investigação (CSI de Taubaté e CS Vila das Graças), guardam sua identidade de saúde pública; a única unidade previdenciária da cidade (agência do INAMPS) caracteriza-se como policlínica dedicada a atuaçāo médica individual às pessoas, incluindo o componente de perícia para auxílios. Dos dez Postos de Assistência MédicoOdontologica (PAMO) existentes na cidade, destinados a assistência básica em áreas periféricas, inclúram-se o do Parque do Sabará, do Alto de Såo Pedro e do Parque Três Marias; e, de igual

TABELA 1

Distribuição dos individuos sadios quanto ao sexo, nos vários locais de exame

\begin{tabular}{lcccccc}
\hline \multirow{2}{*}{ Sexo } & CS & INAMPS & INPS & PAMO & HE & Total \\
\cline { 2 - 7 } & 1.386 & 2.004 & 112 & 241 & 243 & 3.986 \\
Masculino & 2.277 & 2.596 & 50 & 708 & 356 & 5.987 \\
Feminino & 3.363 & 4.600 & 162 & 949 & 599 & 9.973 \\
\hline Total & &
\end{tabular}

CS: $\quad$ Centro de Saúde

INAMPS: Instituto Nacional de Assistência Médica e Previdência Social

INPS: Instituto Nacional de Previdência Social

PAMO: Posto de Assistência Médico-Odontológica

HE: $\quad$ Hospital-Escola da Faculdade de Medicina de Taubaté

TABELA 2

Distribuiçăo dos indivíduos sadios quanto à faixa etária, segundo sexo e locais de exames

\begin{tabular}{|c|c|c|c|c|c|c|c|c|c|c|c|c|}
\hline \multirow{2}{*}{$\begin{array}{l}\text { Faixa } \\
\text { Etária }\end{array}$} & \multicolumn{6}{|c|}{ Masculino } & \multicolumn{6}{|c|}{ Feminino } \\
\hline & Cs & INAMPS & INPS & PAMO & $\mathrm{HE}$ & Total & CS & INAMPS & INPS & PAMO & $\mathrm{HE}$ & Total \\
\hline $\begin{array}{c}15 \longrightarrow 20 \\
20 \longrightarrow 30 \\
30 \longrightarrow 40 \\
40 \rightarrow 50 \\
50 \longrightarrow 60 \\
60 \longrightarrow 70 \\
>70\end{array}$ & $\begin{array}{r}624 \\
210 \\
138 \\
181 \\
115 \\
75 \\
43\end{array}$ & $\begin{array}{r}230 \\
395 \\
407 \\
404 \\
298 \\
181 \\
89\end{array}$ & $\begin{array}{r}5 \\
13 \\
28 \\
30 \\
19 \\
17 \\
0\end{array}$ & $\begin{array}{l}48 \\
16 \\
32 \\
69 \\
33 \\
26 \\
17\end{array}$ & $\begin{array}{l}20 \\
39 \\
31 \\
54 \\
39 \\
32 \\
28\end{array}$ & $\begin{array}{l}927 \\
673 \\
636 \\
738 \\
504 \\
331 \\
177\end{array}$ & $\begin{array}{r}624 \\
554 \\
333 \\
339 \\
237 \\
112 \\
78\end{array}$ & $\begin{array}{l}285 \\
476 \\
400 \\
521 \\
484 \\
307 \\
123\end{array}$ & $\begin{array}{c}0 \\
3 \\
10 \\
14 \\
9 \\
14 \\
0\end{array}$ & $\begin{array}{c}98 \\
132 \\
130 \\
129 \\
118 \\
68 \\
33\end{array}$ & $\begin{array}{l}46 \\
31 \\
54 \\
77 \\
62 \\
61 \\
16\end{array}$ & $\begin{array}{r}1.053 \\
1.205 \\
927 \\
1.080 \\
910 \\
562 \\
250\end{array}$ \\
\hline Total & 1.386 & 2.004 & 112 & 241 & 243 & 3.986 & 2.277 & 2.596 & 50 & 708 & 356 & 5.987 \\
\hline
\end{tabular}

CS: $\quad$ Centro de Saúde

INAMPS: Instituto Nacional de Assistência Médica e Previdência Social

INPS: Instituto Nacional de Previdência Social

PAMO: Posto de Assistência Médico-Odontológica

HE: Hospital-Escola da Faculdade de Medicina de Taubaté 
sorte, Hospital-Escola da Faculdade de Medicina de Taubaté.

As informaçóes clínicas foram todas coletadas por um mesmo investigador, (R. C. N), classificadas segundo Madrid ${ }^{1}$ (1953) e registradas no ins-

\section{TABELA 3}

Distribuição dos individuos sadios, segundo faixa etária e sexo

\begin{tabular}{lrrr}
\hline $\begin{array}{l}\text { Faixa } \\
\text { Etária }\end{array}$ & Masculino & Feminino & Total \\
\hline $15-120$ & 927 & 1.053 & 1.980 \\
$20-125$ & 375 & 648 & 1.023 \\
$25-30$ & 298 & 557 & 855 \\
$30-135$ & 321 & 454 & 775 \\
$35-40$ & 315 & 473 & 788 \\
$40-45$ & 301 & 463 & 764 \\
$45-150$ & 437 & 617 & 1.054 \\
$50-55$ & 257 & 436 & 693 \\
$55-160$ & 247 & 474 & 721 \\
$60-65$ & 194 & 330 & 524 \\
$65-70$ & 137 & 232 & 369 \\
$70-75$ & 96 & 148 & 244 \\
$75-180$ & 57 & 70 & 127 \\
$80-185$ & 19 & 24 & 43 \\
$85-90$ & 3 & 8 & 11 \\
$90-95$ & 2 & 0 & 2 \\
\hline Total & 3.986 & 5.987 & 9.973 \\
\hline
\end{tabular}

trumento de pesquisa. $O$ acompanhamento periodico dos procedimentos foi executado trimestralmente pelos outros autores, que atuaram, portanto, como consultores externos (auditores independentes em duplo controle).

Os resultados dos exames clínicos e os dados pessoais dos indivíduos indicados para participarem do projeto foram armazenados em disquetes MD 550-01 - dupla face. Para cada um dos sexos, foi construido um arquivo de dados subdividido em dois subarquivos, um para os sadios e outro para os portadores da enfermidade. Esses subarquivos contêm um número que identifica a unidade amostrada, a unidade assistencial e a clínica de origem, 0 nome do indivíduo, a idade e a data do exame. No caso de portadores de hanseníase, complementa-se com a forma clínica e a existência ou năo de leső́es dermatológicas e neurológicas.

\section{RESULTADOS}

Foram coletadas 10.013 unidades de observaçסes, entre sadios e doentes detectados.

Destas, em 57 se processou a suspeita diagnóstica de hanseníase, sendo uma, ao final, caracterizada como portadora de meralgia parestésica e outra como monoreurite cubital; quinze trataram-se da moléstia, encontrando-se âtualmente já em alta; quarenta remanesceram, caracterizadas, pois, como doentes em atividade.

Os resultados obtidos estão apresentados nas Tabelas 1 a 6.

TABELA 4

Distribuição dos indivíduos sadios quanto ao tipo de clínica, segundo sexo e local de atendimento

\begin{tabular}{|c|c|c|c|c|c|c|c|c|c|c|c|c|}
\hline \multirow{2}{*}{ Clínica } & \multicolumn{6}{|c|}{ Masculino } & \multicolumn{6}{|c|}{ Feminino } \\
\hline & cs & INAMPS & INPS & PAMO & HE & Total & cs & INAMPS & INPS & PAMO & HE & Total \\
\hline $\begin{array}{l}\text { Cart. Saude } \\
\text { Oftalmologia } \\
\text { Otorrinologia } \\
\text { Neurologia } \\
\text { C. Médica } \\
\text { C. Cirúrgica } \\
\text { Ortopedia } \\
\text { Gineo e Obst. } \\
\text { C. Geral } \\
\text { Pericia Médica }\end{array}$ & $\begin{array}{r}889 \\
246 \\
- \\
98 \\
\vdots \\
153\end{array}$ & $\begin{array}{r}- \\
: \\
761 \\
204 \\
512 \\
376 \\
151\end{array}$ & $\begin{array}{r}: \\
\vdots \\
\vdots \\
\vdots \\
112\end{array}$ & $\begin{array}{r}: \\
: \\
\vdots \\
\vdots \\
241\end{array}$ & $\begin{array}{r}: \\
3 \\
3 \\
162 \\
75 \\
\vdots \\
\vdots \\
-\end{array}$ & $\begin{array}{r}889 \\
246 \\
3 \\
3 \\
3 \\
1.021 \\
279 \\
512 \\
770 \\
263\end{array}$ & $\begin{array}{r}618 \\
422 \\
- \\
135 \\
\\
454 \\
648\end{array}$ & $\begin{array}{r}: \\
\vdots \\
1.214 \\
179 \\
198 \\
295 \\
632 \\
78\end{array}$ & $\begin{array}{l}: \\
: \\
: \\
: \\
: \\
50\end{array}$ & $\begin{array}{c}: \\
\vdots \\
\vdots \\
708\end{array}$ & $\begin{array}{r}- \\
1 \\
4 \\
282 \\
67 \\
\dot{2} \\
-\end{array}$ & $\begin{array}{r}618 \\
422 \\
1 \\
4 \\
1.631 \\
246 \\
198 \\
751 \\
1.988 \\
128\end{array}$ \\
\hline Total & 1.386 & 2.004 & 112 & 241 & 243 & 3.986 & 2.277 & 2.596 & 50 & 708 & 356 & 5.987 \\
\hline
\end{tabular}

CS: Centro de Saúde

INAMPS: Instituto Nacional de Assistência Médica e Previdência Social

INPS: Instituto Nacional de Previdência Social

PAMO: Pcato de Assistência Médico-Odontológica

HE: Hospital-Escola da Faculdade de Medicina de Taubaté 


\section{TABELA 5}

Distribuiçăo do coeficiente de prevalência para a cidade de Taubaté em 1987, por faixa etária/sexo, com destaque para o número de casos esperados e observados

\begin{tabular}{|c|c|c|c|c|c|c|}
\hline $\begin{array}{c}\text { Faixa Etária/ } \\
\text { Sexo }\end{array}$ & Populaçăo & Doentes & $\begin{array}{c}\text { Coeficiente } \\
(\%,)\end{array}$ & $\begin{array}{l}\text { Tamanho } \\
\text { Amostral }\end{array}$ & $\begin{array}{c}\text { Casos } \\
\text { Esperados }\end{array}$ & $\begin{array}{c}\text { Casos } \\
\text { Observados }\end{array}$ \\
\hline $\begin{array}{l}15-20(\mathrm{M}) \\
15-20(\mathrm{~F}) \\
20-25 \mathrm{M}) \\
20-25(\mathrm{~F}) \\
25-30 \mathrm{M}) \\
25-30(\mathrm{~F}) \\
30-35 \mathrm{M}) \\
20-35(\mathrm{~F}) \\
35-40(\mathrm{M}) \\
35-40(\mathrm{~F}) \\
40-50 \mathrm{M}) \\
40-50(\mathrm{~F}) \\
50-60(\mathrm{M}) \\
50-60(\mathrm{~F}) \\
60-70(\mathrm{M}) \\
60-70(\mathrm{~F}) \\
>70(\mathrm{M}) \\
>70(\mathrm{~F})\end{array}$ & $\begin{array}{r}10.287 \\
10.534 \\
10.475 \\
10.297 \\
10.039 \\
9.478 \\
9.125 \\
8.660 \\
6.880 \\
6.789 \\
9.814 \\
9.815 \\
6.376 \\
6.906 \\
3.796 \\
4.627 \\
2.477 \\
2.306\end{array}$ & $\begin{array}{r}3 \\
6 \\
16 \\
9 \\
22 \\
10 \\
23 \\
15 \\
14 \\
15 \\
43 \\
23 \\
24 \\
24 \\
27 \\
20 \\
7 \\
6\end{array}$ & $\begin{array}{l}0,2916 \\
0,5696 \\
1,5275 \\
0,8740 \\
2,1915 \\
1,0551 \\
2,5206 \\
1,7321 \\
2,0349 \\
2,2095 \\
4,3815 \\
2,3434 \\
3,7641 \\
3,4752 \\
7,1128 \\
4,3225 \\
2,8260 \\
2,6019\end{array}$ & $\begin{array}{r}927 \\
1.053 \\
375 \\
648 \\
298 \\
557 \\
321 \\
454 \\
315 \\
473 \\
738 \\
1.080 \\
504 \\
910 \\
331 \\
562 \\
177 \\
250\end{array}$ & $\begin{array}{l}0,27 \\
0,60 \\
0,57 \\
0,57 \\
0,65 \\
0,59 \\
0,81 \\
0,79 \\
0,64 \\
1,05 \\
3,23 \\
2,53 \\
1,90 \\
3,16 \\
2,35 \\
2,43 \\
0,50 \\
0,65\end{array}$ & $\begin{array}{l}3 \\
2 \\
2 \\
1 \\
1 \\
0 \\
1 \\
0 \\
3 \\
3 \\
6 \\
5 \\
6 \\
2 \\
3 \\
2 \\
0 \\
0\end{array}$ \\
\hline $\begin{array}{l}\text { Masculino } \\
\text { Feminino } \\
\text { Total } \\
\text { Pop. Geral }\end{array}$ & $\begin{array}{r}69.269 \\
69.412 \\
138.681 \\
209.256\end{array}$ & $\begin{array}{l}179 \\
128 \\
307 \\
311\end{array}$ & $\begin{array}{r}2,5841 \\
1,8441 \\
2,2137 \\
-\end{array}$ & $\begin{array}{r}3.986 \\
5.987 \\
9.973 \\
-\end{array}$ & $\begin{array}{r}10,30 \\
11,04 \\
22,08 \\
-\end{array}$ & $\begin{array}{l}25 \\
15 \\
40 \\
-\end{array}$ \\
\hline
\end{tabular}

Taxa de prevalência: $3,99 / 1000$

Intervalo de confiança: $3,365 / 1000 \leq \mathrm{p} \leq 4,625 / 1000$

Acréscimo minimo de $52 \%$

Acréscimo máximo de $109 \%$

TABELA 6

Distribuição de doentes detectados por forma clínica, segundo condiçăo do doente registrado ou caso novo

Forma

Condiçăo

\begin{tabular}{|c|c|c|c|c|c|c|}
\hline \multirow[t]{2}{*}{ Clínica } & \multicolumn{2}{|c|}{ Doente Registrado } & \multicolumn{2}{|c|}{ Caso Novo } & \multicolumn{2}{|c|}{ Total } \\
\hline & Freqüência & $\%$ & Freqüuência & $\%$ & Frequuência & $\%$ \\
\hline Indeterminada & 1 & 5,9 & 13 & 56,6 & 14 & 35,0 \\
\hline Tuberculbide & 4 & 23,5 & 4 & 17,4 & 8 & 20,0 \\
\hline Dimorfa & 2 & 11,8 & 3 & 13,0 & 5 & 12,5 \\
\hline Virchowiana & 10 & 58,8 & 3 & 13,0 & 13 & 32,5 \\
\hline Total & 17 & 100,0 & 23 & 100,0 & 40 & 100,0 \\
\hline
\end{tabular}

\section{DISCUSSÃO}

Em relação aos examinados indenes clinicamente à moléstia, da Tabela 1 , depreende-se o predomínio dos elementos de sexo feminino sobre os de sexo masculino, fato conhecido nos registros das agências de saúde em relação a morbilidade, ao contrário do observado quanto à mortalidade, onde esta proporção se inverte (Gonçalves e Gonçalves ${ }^{11}$, 1988). Nas Tabelas 2 e 3, observa-se compatível predominância de adultos jovens, em ambos os sexos, em relaçăo às faixas etárias extremas; na Ta- 
bela 4 , confirma-se a já referida ainda existente especificidade das agências de saúde segundo suas origens, assim é, por exemplo, que os exames médicos para carteira de saúde ocorreram apenas em CS, bem como a perícia médica só se dá na unidade previdenciária.

Quanto aos doentes detectados, os 40 confirmados, em total de 10.013 indivíduos examinados, significam uma taxa de prevalência de 3,99/1000, com intervalo de confiança (ao nível de $95 \%$ de confiabilidade) variando de 3,365 a 4,625/1000, a qual indica que o acréscimo mínimo estimado é da ordem de $52 \%$ e o máximo de $109 \%$.

Considerando-se que o serviço de controle da regiåo vem sendo admitido, pelos padrőes correntes em nosso meio, como satisfatório*, o valor superior do intervalo de confiança da diferencial aqui obtida na taxa de prevalência é superior até à estimativa referida de Bechelli e Martinez Dominguez ${ }^{3}$ (1966), que, como visto, é a mais elevada das mencionadas (Noordeen e Lopes Bravo ${ }^{18}, 1986$ e Lechat ${ }^{12}, 1983$ ).

Tendo-se presente que o denominador aqui utilizado não é a populaçăo geral, mas pessoas adstritas às unidades sanitárias, que as demandaram no período de estudo, supreende-se a base do "iceberg" com maior amplitude do que o estimado pelos indicadores utilizados pelos especialistas da Organizaçăo Mundial da Saúde. Recorde-se, no entanto, "que os valores para estas estimativas relacionavam-se a toda a população e não apenas a pessoas de quinze ou mais anos: a adoçăo deste último critério leva a um aumento do índice, enquanto a do primeiro induz a uma diminuição" (Bechelli, 1988)**.

Tais achados lembram relatos especializados não infreqüentes: na cidade mineira de Candeias, por exemplo, durante doze anos (de 06.12.31 a 20.02.43) foram fichados seis doentes, enquanto durante um censo extensivo, em apenas cinco semanas (de 21.02.41 a 26.03.41), detectaram-se 46 (Del Favero 7 1953); mais expressivo ainda foi o número de doentes despistados, 87, a seguir, durante o censo intensivo; recorde-se, a respeito, que a população geral da cidade era, à época, de 14.002 habitantes, dos quais 11.305, da zona rural (Del Fave$\left.10^{6}, 1948\right)$.

$\mathrm{Na}$ estratificação por faixas etárias (Tabela 5), o posto mais freqüente nas distribuiçōes tanto de valores observados quanto esperados é o de homens na faixa etária 40-50 anos; quantitativamente, por outro lado, a proporcionalidade do di- ferencial de acréscimo revela flutuaçōes, sendo exatamente esse posto um exemplo expressivo; observam-se 6 quando esperar-se-iam 3.

Em relação à proporçăo sexual observada, registra-se predomínio de homens doentes sobre mulheres. Ademais, algumas proporçoes observadas neste grupo de enfermos merecem destaque. Assim é que dos detectados, $41,1 \%$ já eram registrados. Em que pese a limitação de comparaçðes, pela diversidade metodológica e variabilidade epidemiológica, rendimentos inferiores têm sido verificados em estudos censitários casa-a-casa, como o relato por Saylon e Aytekin ${ }^{24}$ (1986); num total de 53 casos detectados, 35 já eram previamente registrados.

O predomínio aparente da forma indeterminada entre os casos novos despistados pelo projeto parece merecer apreciação destacada. Originalmente sempre entendia-se esta proporção como indicativa de programas de controle ágeis e eficientes para surpreender os casos novos ainda em sua fase inicial (Nobrega 17, 1981). No entanto, à medida que se aprofundaram os conhecimentos nosográficos, inclusive pelos estudiosos anglosaxônicos(Petit 22, 1981), foi-se admitindo que a forma indeterminada poderia apresentar duração fugaz, inclusive não superior a um ano. Corroborativamente, fatos de diferentes origens foram se acumulando. Gonçalves e col. ${ }^{12}$ (1984), revendo clinicamente 88 casos novos de tal forma no Distrito Federal, puderam confirmar apenas 22, Pino e col. ${ }^{23}$ (1983) chegaram às mesmas conclusర̃es, confrontando a clínica com a histologia de quase 500 casos. Assim é que, recentemente, os indicadores epidemiológicos básicos para a vigilância da luta contra a hanseníase (Lechat e Vanderveken ${ }^{14}, 1984$ ) incluem, para a realidade brasileira, não a proporção de casos novos da forma indeterminada sobre as demais, mas a proporção de casos novos da tuberculóide em relação aos casos novos virchowianos, dimorfos e tuberculóides (Mello e col. ${ }^{16}$, 1984).

Essa fugacidade não indicaria, no entanto, apenas evolução no sentido de polaridade precoce, mas, também, no de regressăo clínica. Tratar-seiam, neste caso, de pessoas-negativas que fariam o quadro clínico, se positivariam e, então, reverteriam. Vale dizer, não fosse o inquérito exploratório, não seriam detectados. Tal hipótese se compatibiliza com a afirmação de Noussitou ${ }^{19}(1976)$, em relação ao comportamento da doença em crianças; as lesōes iniciais regridem espontaneamente, sem tratamento, em aproximadamente $75 \%$ dos casos".

\footnotetext{
- Embora se possa interrogar, se, pelas dificuldades atuais da operacionalizaçăo de serviços especidalizados em saúde pública em nosso meio, o programa de vigilância de contactos e de deteç̧ăo de casos é satisfatório ou apenas regular.

* Comunicação pessoal.
} 


\section{AGRADECIMENTOS}

Expressa-se publicamente o reconhecimento e dívida intelectual para com as valiosas contribuiçðes decorrentes das leituras críticas dos originais pelo Prof. Dr. Luiz Marino Bechelli, Prof. Ca- tedrático de Dermatologia da Faculdade de Medicina de Ribeirăo Preto da Universidade de São Paulo e pelo Dr. Jair Ferreira, Chefe da Equipe de Dermatologia Sanitária da Secretaria Estadual de Saúde e Meio Ambiente do Rio Grande do Sul.

OPROMOLLA, D. V. et al. (An estimate of the prevalence of hanseniasis by means of investigation into non-specific demand for health services]. Rev. Saúde públ., S. Paulo, 24: 178-85, 1990.

ABSTRACT: In view of the importance of knowing the prevalence and incidence rates of a disease to learn about its behavior and control at the collective level, a study was undertaken to determine the occurrence of hanseniasis among the clients of health agencies and to expore the use of this methodology for estimating the epidemiologic "iceberg" of the disease, i. e., the total number of cases including those that are not officially reported. The city of Taubaté in the Paraiba Valley, State of S. Paulo, Brazil, was chosen for the study. All clients aged 15 years or older were screened regardless of variables such as sex, age, soctal condition or marital status. The study was based on what is known about the populational distribution of the disease and the characteristics of health services. In view of the local peculiarities of operationalization (e.g., identity of the different clientelles, availability of offices, hours of greatest flux), the sampling process used was simple randomization. The patients with active disease detected, 40 of the 10,013 persons examined, correspond to a prevalence of $3.99 / 1000$, whith a confidence interval (at the 5\% level of reliability) of 3,365 to $4,625 / 1000$, indicating that the minimum estimated increase of prevalence is of the order of $52 \%$ and the maximum estimated increase is of the order of $109 \%$. The indeterminate form of the disease predominated among the patients $(35.00 \%)$, and this predominance was even more explicit when the patients were classified as registered or new cases: in this latter category, the indeterminate form reached $56.53 \%$ and its distribution by age range was close to that observed in the officially recorded data, as verified by comparing observed cases with expected cases calculated from population indicators. As to sex ratio, a was predominance of males was observed.

KEYWORDS: Leprosy, epidemiology. Health services.

\section{REFERENCIAS BIBLIOGRÁFICAS}

1. BASOMBRIO, G.; PRIETO, J. G.; KITAMURA, K.; RABELLO, S. E. A.; WADE, H. W.; COCHRAINE, R. G.; KHANOLKAR, V. R.; LATAPI, S.; VEGAS, M.; ARNOLD, H. Draft report of classification committee. In: Congreso Internacional de Leprologia, $6^{\circ}$, Madrid, 1953. Memoria. Madrid, Gobiemo de España. Asociacion Internacional de la Lepra, 1953. p. 75-8.

2. BECHELLI, L. M. \& CURBAN, G. V. Compêndio de dermatologia. São Paulo, Atheneu Ed., 1988.

3. BECHELLI, L. M. \& MARTINEZ DOMINGUEZ, V. Proposed methods for estimating leprosy prevalence. Bull. Wld Hith Org., 34: 811-26, 1966.

4. BECHELII, L. M.; GALLEGO GARBAJOSA, P.; GYI, M. M.; VEMURA, K.; SUNDARESAN, T.; TAMONDONG, G.; MARTINEZ DOMINGUES, V.; SANSARRIQ, H.; WALTER, J. Proposed method for estimating leprosy prevalence based on rates in children. Bul. Org. mond. Santé, 48: 502-3, 1973.

5. COCHRAN, W. G. Técnicas de amostragem. São Paulo, Fundo Cultura, 1965.

6. DEL FAVERO, W. O censo intensivo de Candeias. Arch. Serv. Nac. Lepra, 6: 87-235, 1948.

7. DEL FAVERO, W. "Follow up" de censo intensivo de Candeias. Bol. Serv. Nac. Lepra, 12: 119-30, 1953
8. FINE, P. E. M. Problems in the collection and analysis of data in leprosy studies. Leprosy Rev., 52 (Suppl. 1): 197-206, 1981.

9. GANAPATI, R. Leprosy control in urban community. In: Intemational Workshop on Leprosy Control in Asia, 5th, Tokio, 1983. Proceedings. Tokyo, Sasakawa Mml. Health Foundation, 1983. p. 13-20.

10. GONÇALVES, A. Aspectos de epidemiologia e controle da hanseníase no Brasil. Bol. Ofic. sanit. panamer., 102: 246-57, 1987.

11. GONÇALVES, A. \& GONÇALVES, M. N. S. Saúde e doença: conceitos básicos. Rev. bras. Ciênc. e Mov., 2(2): 48-56, 1988.

12. GONCALVES, A.; RIBEIRO, M. A. C. R.; OPROMOLLA, D. V. A.; PADOVANI, C. R.; CONSORTE, J.; BELINI, J. M.; CONÇALVES, N. N. S. Dermatoglyphs in leprosy: generation of a linear discriminating function viewing the identification of groups at risk. In: Intemational Leprosy Congress, 12th, New Delhi, 1984. Abstracts. New Delhi, 1984. p. 405.

13. LECHAT, M. F. Leprosy in a global context. In: International Workshop on Leprosy Control in Asia, $5^{\text {th }}$, Tokyo, 1983. Proceedings. Tokyo, Sasakawa Mml. Health Foundation, 1983. p. 8-12. 
14. LECHAT, M. F. \& VANDERVEKEN, M. Indicadores epidemiológicos búsicos para la vigilancia de la lucha contra la lepra. Washington, D. C., Organización Panamericana de la Salud, 1984.

15. LECHAT, M. F.; VANDERVEKEN, M.; DECLERQ, E.; MISSION, C. B. Incidence de la lepre: analyse des tendances. Wld Hlth Statist. Quart., 39: 128-37, 1986.

16. MELLO, A.; GONCALVES, A.; ROMERO, A. B.; DINIS FILHO, C.; FERREIRA, J.; CUNHA, M. G. S.; OLIVEIRA, M. L. W.; RIBEIRO, M. A. C. L.; ALEGRE, R. M.; TARLE, S. F.; RODRIGUES, S. S. F.; LEVY, S. N. Relatb́rio da reunido do grupo assesssor em registro de dados. Rio de Janeiro, Divisăo Nacional de Dermatologia Sanitária, 1984.

17. NOBREGA, E. C. Endemia hansênica: Brasil - 1979. Brasilia, Divisăo Nacional de Dermatologia Sanitária, 1981.

18. NOORDEEN, S. LOPEZ BRAVO, L La lepre dans le monde. Rapp. trimest. Statist. sanit. mond., 39: 122-8, 1986.

19. NOUSSITOU, F. M. Lepra infantil. Ginebra, Organización Mundial de la Salud, 1976.

20. OPROMOLlA, D. V. A.; NOBREGA, R. C.; GONÇALVES, N. N. S.; PADOVANI, C. R.; GONÇALVES, A. Estudo da prevalência da han- seníase em clientela da agência de saúde; Relatório técnico do CNPq. Bauru, 1988.

21. ORGANIZAÇĀo PAN-AMERICANA DA SAÚdE. Manual para o controle da hansentase. Washington, D. C., 1983.

22. PETIT, J. M. S. Should indeterminate leprosy ever be diagnosed? Int. J. Leprosy, 49: 95-6, 1981.

23. PINO, G. S.; FERREIRA, J.; BERNARDI, C.; PONZIO, H. A. S.; PONZIO, T. C. Evaluacion clínje e histologica de 417 casos con el diagnostico de lepra. Med. cut., 9:99-106, 1983.

24. SAYLON, T. \& AYTEKIN, A. K. Mass screening in leprosy endemic areas of Turkey: preliminary report. Leprosy Rev., 57: 243-9, 1986.

25. SOMSE, P.; TESTA, J.; TROTOBAS, M.; CABON, M.; LIMBASSA, J.; BAQUILLON, G. Evaluation de l'endémie lépreuse dans la préfecture de la Haute; Sangha in République Centrafricaine, au cours d' une enquête: sondage effectus au mois d'soût, 1983. Acta leprol., 3: 85-92, 1985.

26. SUNDARESAN, T. K. \& SANSARRICQ, M. Sample surveys in leprosy: an introductory manual. Geneva, Draft 1982.

Recebido para publicação em 15/8/1989 Aprovado para publicação em 06/3/1990. 